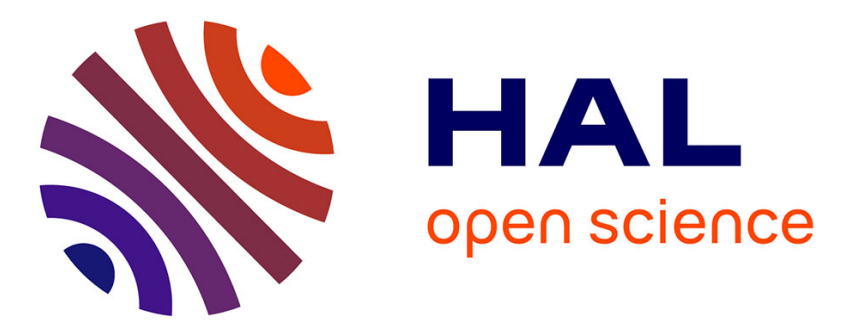

\title{
Using location services to autonomously drive flying mobile sinks in Wireless Sensor Networks
}

Nicola Roberto Zema, Nathalie Mitton, Giuseppe Ruggeri

\section{To cite this version:}

Nicola Roberto Zema, Nathalie Mitton, Giuseppe Ruggeri. Using location services to autonomously drive flying mobile sinks in Wireless Sensor Networks. International Conference on Ad Hoc Networks (AdHocNets), Aug 2014, Rhodes, Greece. hal-01058259

\section{HAL Id: hal-01058259 \\ https://hal.inria.fr/hal-01058259}

Submitted on 26 Aug 2014

HAL is a multi-disciplinary open access archive for the deposit and dissemination of scientific research documents, whether they are published or not. The documents may come from teaching and research institutions in France or abroad, or from public or private research centers.
L'archive ouverte pluridisciplinaire HAL, est destinée au dépôt et à la diffusion de documents scientifiques de niveau recherche, publiés ou non, émanant des établissements d'enseignement et de recherche français ou étrangers, des laboratoires publics ou privés. 


\title{
Using location services to autonomously drive flying mobile sinks in Wireless Sensor Networks
}

\author{
Nicola Roberto Zema ${ }^{2}{ }^{\star}$, Nathalie Mitton ${ }^{1}$, and Giuseppe Ruggeri ${ }^{2}$ \\ 1 Inria \\ nathalie.mitton@inria.fr \\ 2 University "Mediterranea" of Reggio Calabria (Italy) \\ \{giuseppe.ruggeri, nicola.zema\}@unirc.it
}

\begin{abstract}
The use of mobility in a Wireless Sensor Network has already been indicated as a feature whose exploitation would increase the performances and the ease of mantainance in these environments. Expecially in a event-based WSN, where is necessary a prompt response in terms of data processing and offloading, a set of mobile flying sinks could be a good option for the role of autonomous data collectors. For those reasons in this paper we propose a distributed algorithm to independently and autonomously drive a mobile sink through the nodes of a WSN and we show its preferability over more classical routing approaches expecially in the presence of a localized generation of large amount of information. Our result shows that, in the case of fairly complete coverage of the area where the nodes lie, it is possible to promptly notify a mobile sink about the presence of data to offload, drive it to the interested area and achieve interesting performances.
\end{abstract}

Key words: Controlled Mobility, Sensor networks, Network scalability and capacity, Network architectural and protocol design.

\section{Introduction}

The delivery and diffusion of precious data in a multihop environment like the one of the Wireless Sensor Networks (WSNs) [29] pose a series of challenges to each of the designer, the maintainer and the user of these specific networks. Energy consumption linked with network lifetime and end-to-end delay are some of the most prominent and it is shown $[11,18,27]$ that applying mobility as a degree of freedom in the network would enhance its performances while easing in the management of the aforementioned challenges. For example, with one or more fixed destinations scattered far apart in the network and with each node as a possible source of multihop traffic, there would be a strong imbalance in

* The research of Nicola Roberto Zema is partially supported by European Union (EU), European Social Fund (ESF), Calabria Local Goverment and Inria Lille. This paper reflects the views only of the authors, and the EU, the ESF, Calabria Local Goverment and Inria Lille cannot be held responsible for any use which may be made of the information contained therein. 
the paths taken by information. This difference would make the nodes around the fixed collectors deplete sooner their batteries and generate rings of unconnected free space around the data sinks [19]. In this situation, using a mobile solution for the collectors leads to balance the load distribution [3]. In reviewed studies, regardless using naive direct [13], multihop[22] or hybrid [27] routing schemes to deliver data to a mobile sink, it is usually supposed that the mobility is uncontrollable or at best predicted. In this paper we propose instead to allow the mobile node to manipulate its path guided by the network itself. We devise the use of a controlled mobility-enhanced sink in a Wireless Sensor Network for data offloading. This peculiar study would research the inferring of the trajectory by a mobile node from the information diffused by the other members of the network. We create an environment in which instead of relying on multihop data diffusion and routing, the nodes of the network will signal to a collector the presence of events associated with the consistent generation of data, like the necessity for a cluster head to unload all the collected values by its neighbor nodes, without requiring an apriori knowledge of its position. The collector will then schedule its movements to reach the interested areas. This is particularly useful in all the environments where a strict temporal response is needed and where large quantities of data are generated. We propose a practical, completely distributed, signaling and movement protocols for a mobile sink and, in support of our design, we present a simulation study where we analyze its performances and make comparisons against more used solutions. Results show that our approach outperform these latters regarding the overall energy expenditure and, up to a certain number of sources, the end-to-end delay. The paper is organized as follows. In Section 2 we overview the existing literature and solutions for the mobility of a sink in a WSN. In Sections 3 and 4 we present our proposal first in its environment and then in its details. Our results are shown in Section 5, while in Section 6 we describe our future proposal and possible research paths.

\section{Related Literature}

Studies regarding maximization of network lifetime in a WSN highlight several key features that need to be addressed apart from budget and coverage requirements. These include energy consumption distribution, energy hole problems and the discovery of efficient routes for data diffusion and dissemination [8, 32]. It is also highlighted that the use of mobility could bring great benefits to the performances and lifetime of a WSN [16, 20, 15]. Bringing mobility in the network in form of a mobile sink shifts the priority of the routing problem. From the optimal and stable finding of efficient routes departing from any point in the network and arriving to a set of fixed sinks, the routing algorithms have to track their movements and try to deliver them the data [28]. The solutions to this problem are various and can be classified using various criteria. Some authors make distinction between the ones that employ the preliminary creation of hierarchical structures and the ones that do not use them [28]. Other authors instead propose the distinction between the routing protocols that use a pure 
multihop approach, the single-hop data collection and hybrid routing schemes [25]. The majority of those approaches however suppose that (i) the mobility of the sinks can at best be predicted and (ii), are tailored for value diffusion rather than multihop flow handling. Regarding the latter issue, there are different works that leverage the use of mobile entities for information gathering and delivering. Data MULES [26, 17] and Message Ferries [31, 23] propose the use of mobile elements to transport large quantities of offloaded data using mobile nodes with enhanced capabilities in respect to the rest of the network. Regarding the former issue, there is a set of works that employs actual controlled mobility for network enhancement. In [4] is proposed a framework comprising of a mathematical optimization, a centralized and distributed heuristic that define a predefined abstract set of movements for a group of mobile sinks improving the expected network lifetime. In [24] is proposed another scheme for the trajectory finding of a mobile sink that uses phased arrays antenna systems to orient itself in a network, given a set of predefined waypoints. Our work is different as we propose an on-demand scheme for sink movement rather than a collection trip, useful in event-driven situation where a prompt response is needed, and as we propose a practical direction finding scheme. We also take as a source the works on position discovery in WSN routing [6] and in particular the research on methods that use geographical properties of the network [7].

\section{Overall Principle and Main Assumption}

The use of the nodes' geographic properties in the design of network protocols is not new and a lot of solutions implement the exploitation of those properties for routing algorithms and data diffusion schemes [11]. However among the limitations highlighted are the scarce efficiency and the energy dispersion inherent in delivering amounts of data not limited to single values. In a sensor network covering an area where the nodes could sense an event, activate themselves and offload the collected data to a sink, that amount is not trivial and it is problematic to deliver it to its destination using the multihop schemes described in literature. Techniques including mobility in the sink help reduce the number of hops to travel for a packet to arrive at destination thus reducing the overall network resource consumption at the expenses of introducing an energy cost for the physical movement. It is pointed out that a good solution would be to use a controlled mobility environment where the sink entity is driven by information given by the network itself [28]. In an event-driven WSN, there would be geographically localized events that make the sensor covering the same area to start collecting environmental information and, possibly, deliver them to temporary storage. In such an environment whenever this localized data is generated and have to be quickly offloaded [9], it is possible for a mobile sink to move directly into the proximity of the temporary collector and reduce to the minimum the number of hops. Also, there would be cases where in addition to the offloading process, there is the need of bringing an external observer in the area of a sensed event even if there is no data to deliver. With these guidelines in mind we first 
devised a protocol for delivering the information about the presence of data to offload in a wireless sensor network and then included it in a controlled mobility framework where the sink can move to the advertised position and collect the data or perform some other local maintenance operations [21]. In our design the trajectory is directly inferred by the network and computed autonomously by the mobile sink. The assumptions we made about our network are that: (i) it is composed by static sensor nodes; (ii) it is dense enough, given the capabilities of a general sensor networking device, to uniformly cover the whole sensing area and (iii) that each node knows its position either because of the presence of a GPS device or because that value has been pre-set. In our proposal, we envision the use of mobile flying robots for the mobile sink role as the possibility to use them as an effective communication station is displayed $[12,14,30]$. In particular, for its capability to hover above a group of sensors waiting for the data offloading to finish, we envisioned the use of quadcopters [5] considering the movement capabilities accordingly.

\section{Supporting Protocol Overview}

Our protocol design takes its roots in the works on geographical data diffusion and in particular the research on the location services. If a node has data to transmit, each time interval $\Delta t_{\text {source }}$ it broadcasts a source advertisement packet containing its ID and its absolute position. Upon reception, the packet is broadcast again only by nodes placed in the band parallel to the north-south absolute axis, whose median passes by the original broadcasting node and whose amplitude is defined by the parameter $\Delta_{\text {width }}$. Also, the nodes that receive a packet, will check against its ID and broadcast it again only after the expiration of a timeout $\Delta t_{\text {source expire }}$. This is possible because the nodes keep track of the various sources of information they come in contact with, in terms of the tuple composed of the ID and the coordinates. In Figure 1 is displayed a working scenario with $\Delta_{\text {width }}$ equal as the node transmission range. The mobile sinks also are capable, upon the expiration of the time interval $\Delta t_{\text {sink }}$, to send sink advertisement packets along a stripe, this time parallel to the east-west absolute axis. Eventually those two stripes will intersect and in the geographical area of superposition there will be nodes that have been reached by both packet types, the shaded gray area of figure 1 . In the case of a single collector, when a node has the information about both the position of the mobile sink and the source, it sends the information about the latter using backtrace packets, which contain the same information as the source advertisement with the addition of the sink ID. Those are broadcasted back to the sink only by the nodes that originally forwarded the sink advertisement packets. Whenever a mobile sink receives the information about a source of traffic it stores the value and, at the expiration of a timer $\Delta t_{\text {movement control }}$, it ranks the sources based on the distance and move to the nearest to initiate the offloading. After the offloading has expired, the mobile sink will check the position of the sources and move again to the new nearest destination if present. In this way it is possible to bypass completely the routing 
of large quantities of packets in an energy constrained multihop environment thus improving the whole performance of the network in terms of end-to-end delays and power consumption.

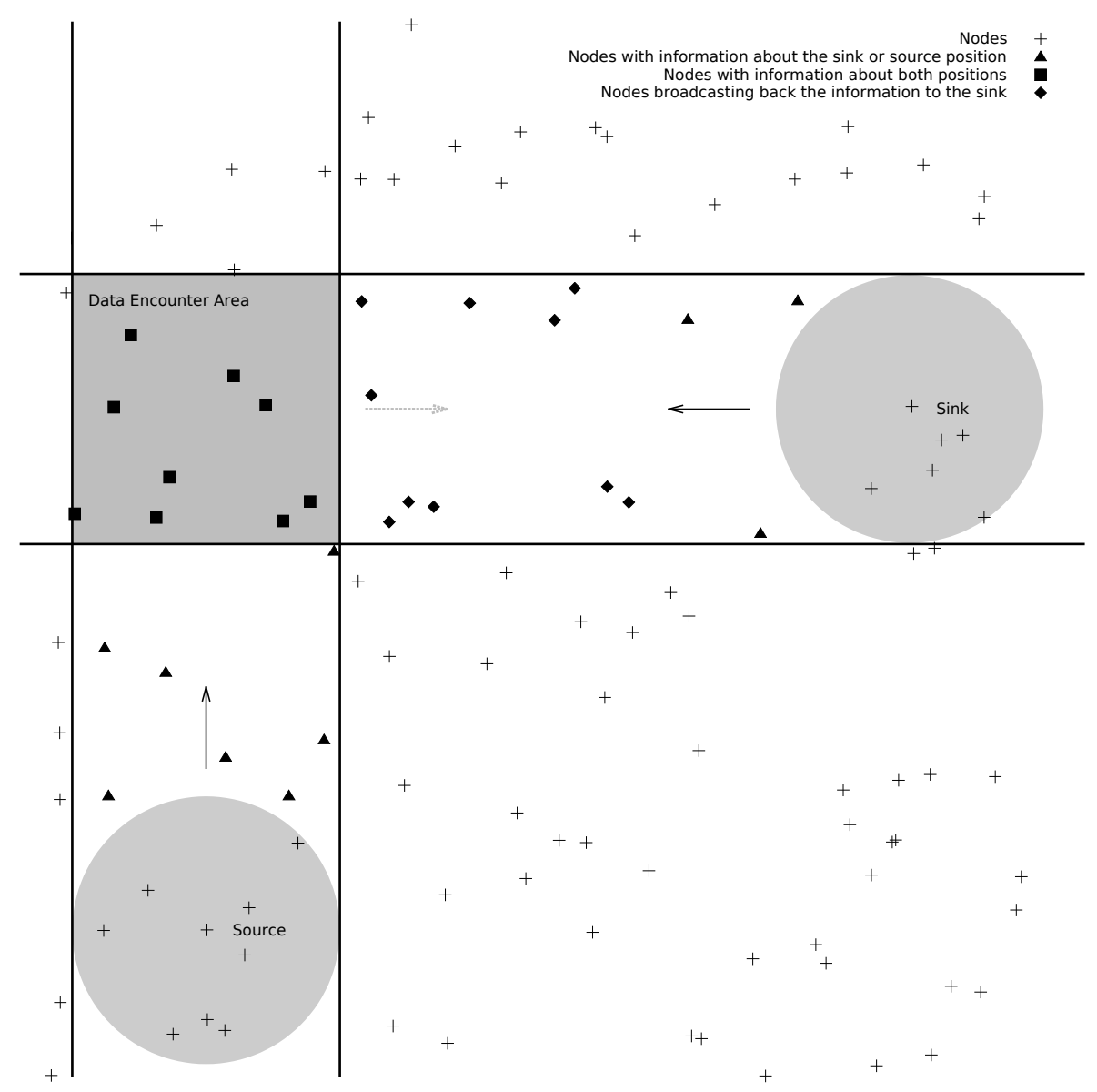

Fig. 1: Example working scenario.

For testing the capability of our proposal to ease the network in cases of large quantities of data to offload, we tested our system against a WSN environment and an implementation of a common solution, the Greedy Geographical Routing.

\section{Highlighted Results}

For our purposes, we set up a network simulation with the wsnet environment $[10,1]$. We defined a topology of 600 uniformly distributed nodes on a 
$500 \mathrm{~m} \times 500 \mathrm{~m}$ area. Each node is equipped with a $2400 \mathrm{MHz}$ bpsk $802.15 .4 \mathrm{com}-$ pliant radio and MAC interface working on a single channel. We set the values of $\Delta t_{\text {source }}, \Delta t_{\text {sink }}$ and $\Delta t_{\text {source expire }}$ as the estimated time necessary for a packet to travel the network diameter with the current nodes' capabilities, while $\Delta_{\text {width }}$ was put as the averaged node's coverage umbrella diameter. The val-

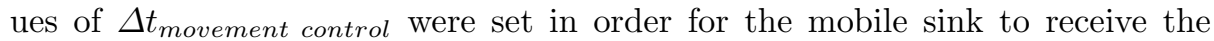
information about the sources and move immediately after the completion of a transfer. In a first set of simulations, for our tests we put a source and a sink at two opposite corners of the topology. We set up a bursty acked application where we made the source transmits a variable size data of $0.25,0.5,1$ and 1.5 $\mathrm{MB}$, representing the data that $\mathrm{s}$ to be offloaded, with the maximum rate and packets size possible for the 802.15.4 MAC [2]. Using our proposal, called Directed Movement in the graphs, the source and the destination will first signal each other and then the sink will move into position to offload the data. For making a comparison, we fed the same bursty traffic to the geographical routing algorithm implemented in stock wsnet [1], that is indicated in the graphs as Greedy Geographic. Each value is the result of a 10 simulative runs whose outputs were processed using a student's t-distributions with $95 \%$ confidence intervals and shown where applicable. Figure 4 shows the time to complete the data offload for various burst sizes, defined as the difference in time between the first packet enqueue in the source and the last received ack. It can be seen that it is dependent of the burst size and it shows a great reduction using our system. The reduction is high enough to suggest the possibility, using our system, to serve multiple sources with ease, especially with large burst size. It is possible to see from Figure 2 that the energy impact on the network is greatly reduced. The figure shows the total number of sent packets in the network, as a sum of application, routing and position signaling traffic where present, by all nodes. Again our proposal, reducing to just one hop the distance the information has to travel make consistent traffic reduction possible thus greatly augmenting the network lifetime. In figure 3 it is shown the ratio between the original application data sent only by the source and destination nodes and the routed, routing and movement traffic where applicable. It can be seen that our system achieves better performances in terms of packets sent and minor time to complete the transfer because it can perform a great reduction in the aforementioned ratio, relying only on our signaling protocol to make the mobile sink reach the sources. In a second set of simulations we tested the capabilities of our system in presence of multiple sources. In the same network topology and environment we put this time up to four sources that had, starting at the same time, to offload $1 \mathrm{MiB}$ of application data to a sink placed in a corner of square area where the nodes were placed. We set up the sources in the other corners and in the center and activate them in the following order during different setups: first the adjacent corners, then the center and then the opposite corner increasing distance. Figure 5 is again a measure of the needed time to complete the transfer comparing our proposal and the greedy geographical routing. Even if in our proposal the time to serve all the sources increases faster than the greedy approach in respect to 
number of sources, it stills outperforms it up to four data generating nodes. It should be noted that, in the same conditions, the total number of sent packets and thus the network energy consumption is not only higher but also increasing faster as the number of sources increases. This aspect of our system can also be seen from figure 6 , where the total traffic is shown. Increasing the sources create a dramatic increase in the packet generation for the greedy geographical approach due to congestion and retransmissions. In the last figure, 7 , a replica of figure 3 for multiple sources, as the total transmitted data increase the ratio between original application data and the routed traffic decreases but our system still achieves better efficiency.

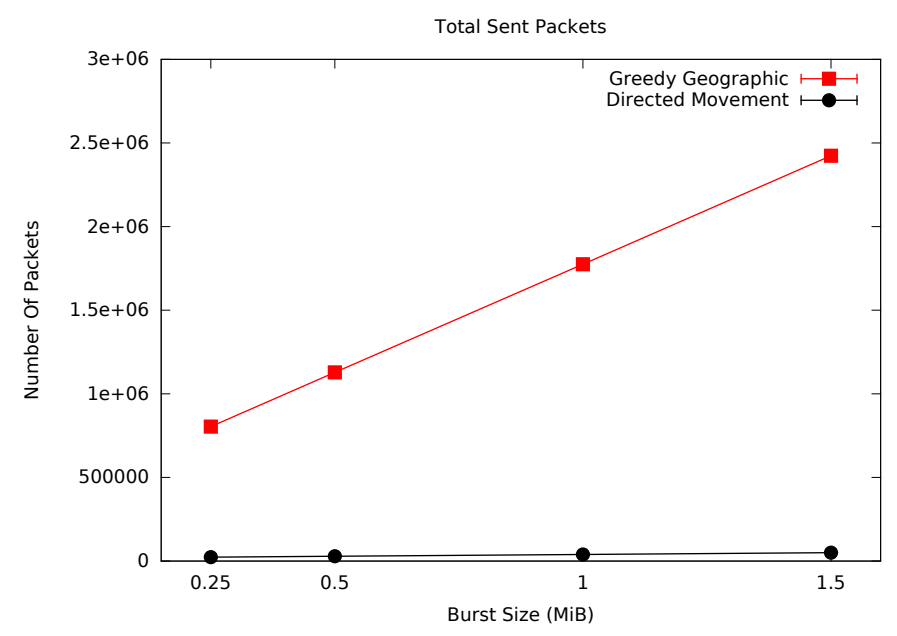

Fig. 2: Total number of sent packets.

\section{Conclusions and possible future research paths}

In this paper we have proposed a practical, completely distributed, signaling and movement protocols for a mobile sink to collect data from advertizing nodes in a wireless sensor network. Our proposal is particularly useful when the quantity of data that needs to be offloaded is not negligible and our event pinpointing system can also be used to drive the mobile nodes towards designated areas where their physical presence is needed. Even if we have shown the feasibility of our driving framework and its advantages against a simple geographical routing system in the reliable delivery of application data, there are still some issues that we plan to address. We suppose that the network topology is dense enough to guarantee the coverage of the geometrical surface where the nodes are placed; else there would be points where the information about the sources and the sinks cannot travel. 


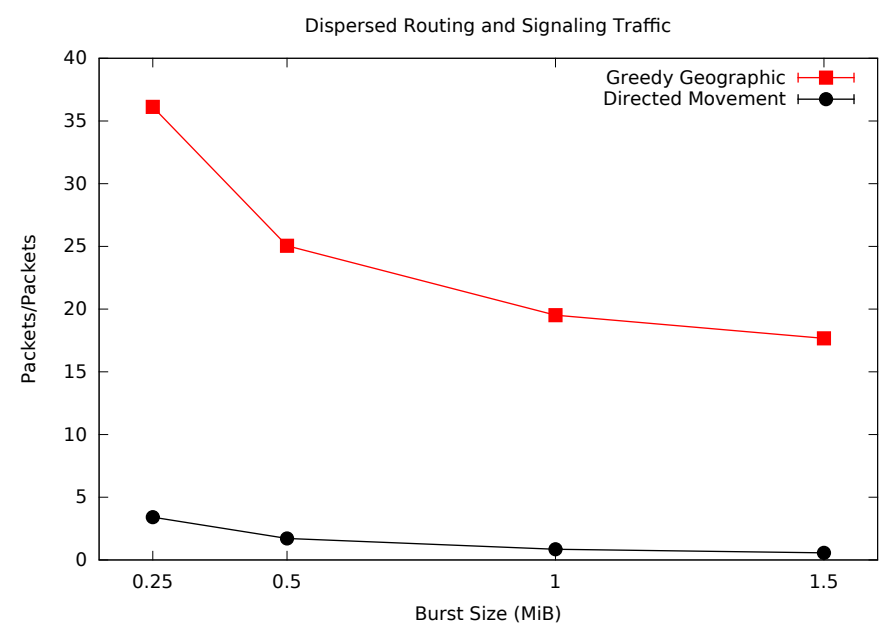

Fig. 3: Ratio between the application data sent by source and destination nodes and the total routing and movement signaling traffic.

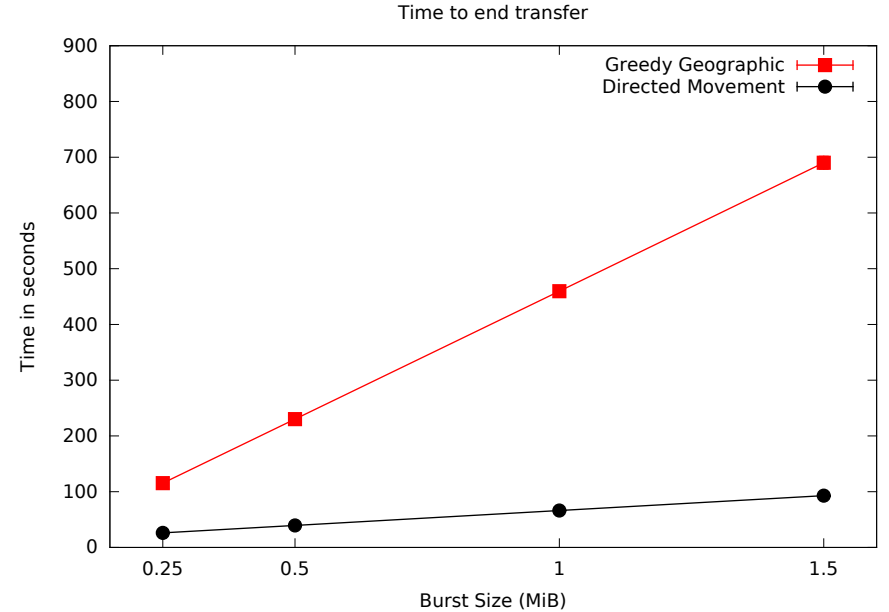

Fig. 4: Time to transfer completion.

It should be devised so an obstacle avoidance system that is capable to make the packets carrying the positions route around coverage holes and continue in the same direction. It should also be analyzed the setup where are present multiple mobile sinks and how to apply to this situation an advanced algorithm that efficiently ranks the various source positions eventually detected by each sink. In this situation would also be necessary a careful tune of the parameters and an accurate evaluation of the mobility inherent cost. Another important research 


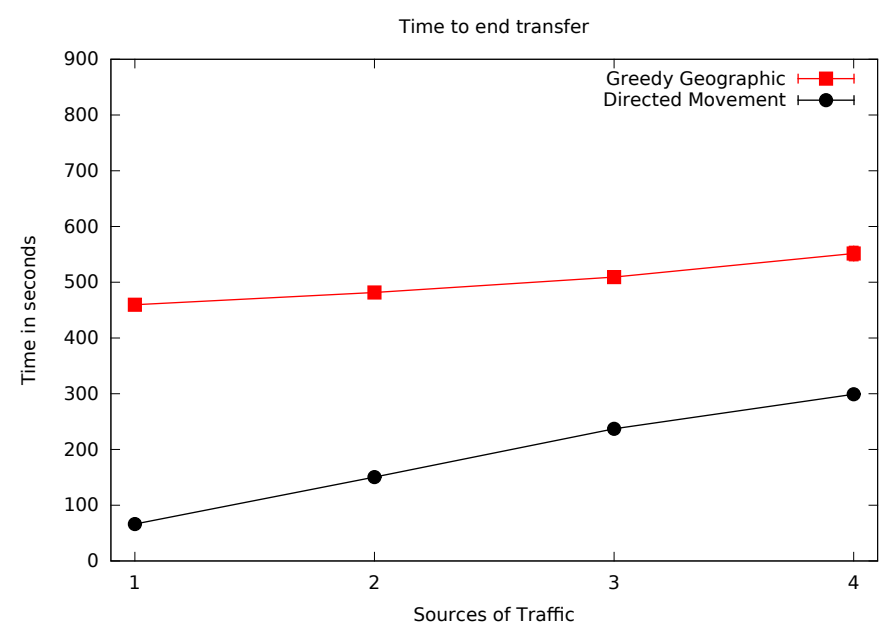

Fig. 5: Time to transfer completion, multiple sources.

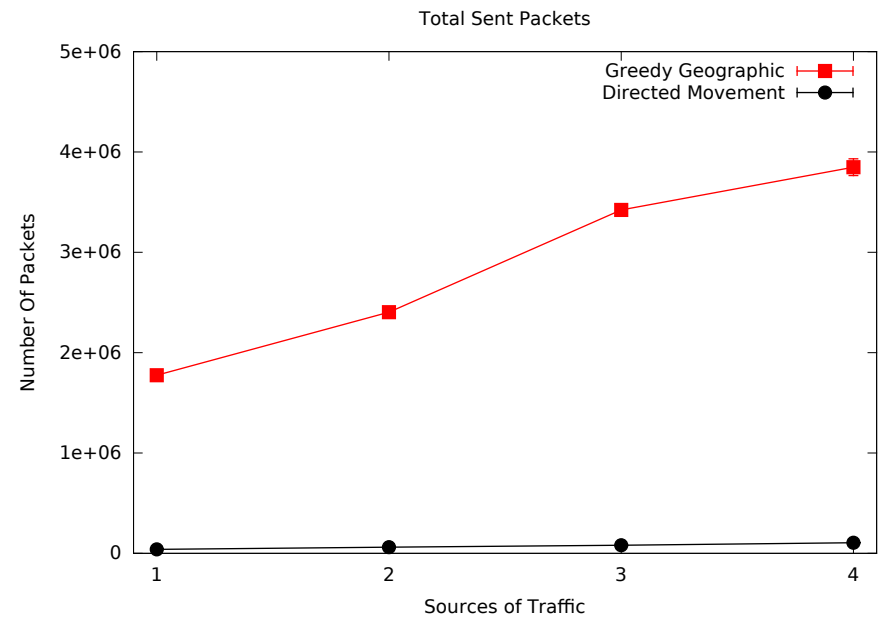

Fig. 6: Total number of sent packets, multiple sources.

direction would be to abandon the dependence on absolute positioning systems and the introduction of a protocol that is capable to swiftly guide the mobile sink through waypoints in the network using only relative positions and inertial navigation, relaxing the assumptions on coordinate awareness.

\section{References}

1. WSNet / Worldsens Simulator - wsnet. available from 


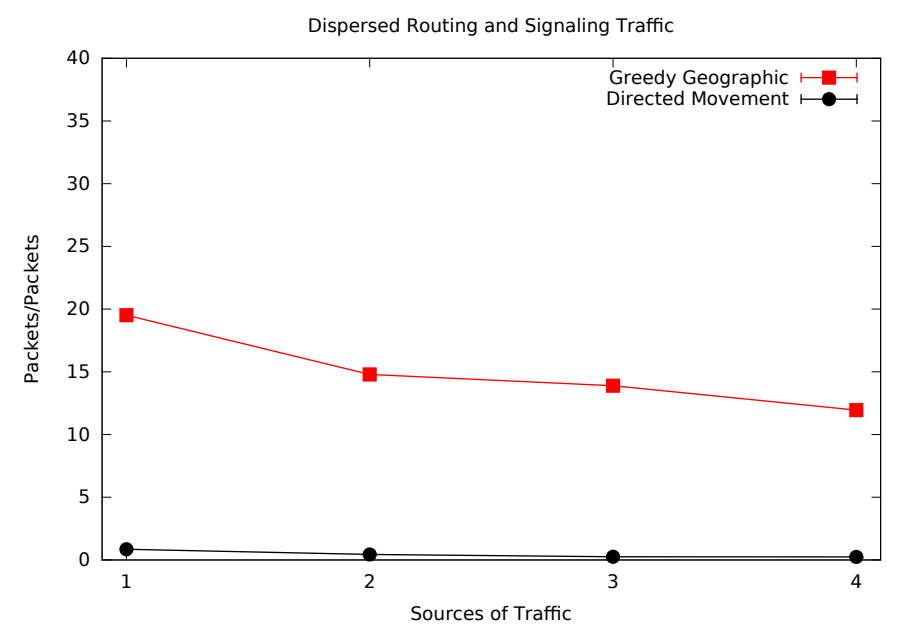

Fig. 7: Ratio between the application data sent by source and destination nodes and the total routing and movement signaling traffic, multiple sources.

http://wsnet.gforge.inria.fr.

2. IEEE Standard for Information Technology- Telecommunications and Information Exchange Between Systems- Local and Metropolitan Area Networks- Specific Requirements Part 15.4: Wireless Medium Access Control (MAC) and Physical Layer (PHY) Specifications for Low-Rate Wireless Personal Area Networks (WPANs). Technical report, 2006.

3. Stefano Basagni, Alessio Carosi, Emanuel Melachrinoudis, Chiara Petrioli, and Z Maria Wang. Controlled sink mobility for prolonging wireless sensor networks lifetime. Wireless Networks, 14(6):831-858, 2008.

4. Stefano Basagni, Alessio Carosi, Chiara Petrioli, and Cynthia A Phillips. Coordinated and controlled mobility of multiple sinks for maximizing the lifetime of wireless sensor networks. Wireless Networks, 17(3):759-778, 2011.

5. Yuanyuan Chen, Jianxin Chen, Liang Zhou, and Yuelin Du. A data gathering approach for wireless sensor network with quadrotor-based mobile sink node. In Ruchuan Wang and Fu Xiao, editors, Advances in Wireless Sensor Networks, volume 334 of Communications in Computer and Information Science, pages 44-56. Springer Berlin Heidelberg, 2013.

6. Wei Cheng, Nan Zhang, Xiuzhen Cheng, Min Song, and Dechang Chen. Timebounded essential localization for wireless sensor networks. IEEE/ACM Transactions on Networking (TON), 21(2):400-412, 2013.

7. S.M. Das, H. Pucha, and Y.C. Hu. Performance comparison of scalable location services for geographic ad hoc routing. In INFOCOM 2005. 24th Annual Joint Conference of the IEEE Computer and Communications Societies. Proceedings IEEE, volume 2, pages 1228-1239 vol. 2, March 2005.

8. M Emre Keskin, İ Kuban Altınel, Necati Aras, and Cem Ersoy. Wireless sensor network lifetime maximization by optimal sensor deployment, activity scheduling, data routing and sink mobility. Ad Hoc Networks, 2014.

9. Luca Filipponi, Andrea Vitaletti, Giada Landi, Vincenzo Memeo, Giorgio Laura, and Paolo Pucci. Smart city: an event driven architecture for monitoring public 
spaces with heterogeneous sensors. In Sensor Technologies and Applications (SENSORCOMM), 2010 Fourth International Conference on, pages 281-286. IEEE, 2010.

10. Antoine Fraboulet, Guillaume Chelius, and Eric Fleury. Worldsens: Development and prototyping tools for application specific wireless sensors networks. In Proceedings of the 6th International Conference on Information Processing in Sensor Networks, IPSN '07, pages 176-185, New York, NY, USA, 2007. ACM.

11. Shuai Gao, Hongke Zhang, and Sajal K Das. Efficient data collection in wireless sensor networks with path-constrained mobile sinks. Mobile Computing, IEEE Transactions on, 10(4):592-608, 2011.

12. Andrea Giorgetti, Matteo Lucchi, Marco Chiani, and Moe Z Win. Throughput per pass for data aggregation from a wireless sensor network via a uav. Aerospace and Electronic Systems, IEEE Transactions on, 47(4):2610-2626, 2011.

13. Yaoyao Gu, Doruk Bozdag, Eylem Ekici, Füsun Özgüner, and Chang-Gun Lee. Partitioning based mobile element scheduling in wireless sensor networks. In SECON, pages 386-395. Citeseer, 2005.

14. Francesca Guerriero, Rosario Surace, Valeria Loscri, and Enrico Natalizio. A multiobjective approach for unmanned aerial vehicle routing problem with soft time windows constraints. Applied Mathematical Modelling, 2013.

15. Elyes Ben Hamida and Guillaume Chelius. Strategies for data dissemination to mobile sinks in wireless sensor networks. Wireless Communications, IEEE, 15(6):3137, 2008.

16. Liang He, Jianping Pan, and Jingdong Xu. A progressive approach to reducing data collection latency in wireless sensor networks with mobile elements. Mobile Computing, IEEE Transactions on, 12(7):1308-1320, 2013.

17. David Jea, Arun Somasundara, and Mani Srivastava. Multiple controlled mobile elements (data mules) for data collection in sensor networks. In Distributed Computing in Sensor Systems, pages 244-257. Springer, 2005.

18. Aman Kansal, Arun A Somasundara, David D Jea, Mani B Srivastava, and Deborah Estrin. Intelligent fluid infrastructure for embedded networks. In Proceedings of the 2nd international conference on Mobile systems, applications, and services, pages 111-124. ACM, 2004.

19. Jian Li and Prasant Mohapatra. Analytical modeling and mitigation techniques for the energy hole problem in sensor networks. Pervasive and Mobile Computing, $3(3): 233-254,2007$.

20. Weifa Liang, Jun Luo, and Xu Xu. Prolonging network lifetime via a controlled mobile sink in wireless sensor networks. In Global Telecommunications Conference (GLOBECOM 2010), 2010 IEEE, pages 1-6. IEEE, 2010.

21. Kalypso Magklara, Dimitrios Zorbas, and Tahiry Razafindralambo. Node discovery and replacement using mobile robot. In Ad Hoc Networks, pages 59-71. Springer, 2013.

22. Ioannis Papadimitriou and Leonidas Georgiadis. Energy-aware routing to maximize lifetime in wireless sensor networks with mobile sink. journal of Communications software and Systems, 2(2):141-151, 2006.

23. Bahadir K Polat, Pushkar Sachdeva, Mostafa H Ammar, and Ellen W Zegura. Message ferries as generalized dominating sets in intermittently connected mobile networks. Pervasive and Mobile Computing, 7(2):189-205, 2011.

24. Jayanthi Rao and Subir Biswas. Network-assisted sink navigation for distributed data gathering: Stability and delay-energy trade-offs. Computer Communications, 33(2):160-175, 2010. 
25. Tifenn Rault, Abdelmadjid Bouabdallah, and Yacine Challal. Wsn lifetime optimization through controlled sink mobility and packet buffering. In Global Information Infrastructure Symposium, 2013, pages 1-6. IEEE, 2013.

26. Rahul C Shah, Sumit Roy, Sushant Jain, and Waylon Brunette. Data mules: Modeling and analysis of a three-tier architecture for sparse sensor networks. Ad Hoc Networks, 1(2):215-233, 2003.

27. Arun A Somasundara, Aman Kansal, David D Jea, Deborah Estrin, and Mani B Srivastava. Controllably mobile infrastructure for low energy embedded networks. Mobile Computing, IEEE Transactions on, 5(8):958-973, 2006.

28. Can Tunca, Sinan Isik, M.Yunus Donmez, and Cem Ersoy. Distributed mobile sink routing for wireless sensor networks: A survey. Communications Surveys Tutorials, IEEE, 16(2):877-897, Second 2014.

29. Jennifer Yick, Biswanath Mukherjee, and Dipak Ghosal. Wireless sensor network survey. Computer networks, 52(12):2292-2330, 2008.

30. Mohamed Younis, Izzet F Senturk, Kemal Akkaya, Sookyoung Lee, and Fatih Senel. Topology management techniques for tolerating node failures in wireless sensor networks: A survey. Computer Networks, 2013.

31. Wenrui Zhao and Mostafa H Ammar. Message ferrying: Proactive routing in highlypartitioned wireless ad hoc networks. In Distributed Computing Systems, 2003. FTDCS 2003. Proceedings. The Ninth IEEE Workshop on Future Trends of, pages 308-314. IEEE, 2003.

32. D. Zorbas, C. Douligeris, and V. Fodor. Target location based sink positioning in wireless sensor networks. In Telecommunications (ICT), 2011 18th International Conference on, pages 21-26, May 2011. 